\title{
Research on the Combination Method of Spare Parts Consumption Data
}

\author{
Shenyang Liu, Qian Zhu, Xiaopei Shi, Yongliang Zhang, Chen Zhu \\ Department of Air Material and Four Stations, Air Force Logistics College, Xuzhou 221000, China
}

Keywords: spare parts consumption data, different environments, combination methods, probability theory

\begin{abstract}
Through an analysis of the problem of lacking of spare parts consumption data in some environments, this paper combines spare parts consumption data in different environments, applies equipment reliability theory and probability theory to establish spare parts consumption combination models. The calculation method could be used to solve the difficult problem of developing a reserve program of spare parts since it is not easy to find out spare parts consumption rule. The models provide a theoretical basis for calculating reserves of spare parts scientifically and have an important guiding significance.
\end{abstract}

\section{INTRODUCTION}

Many scholars have made scientific researches on spare parts consumption ( $\mathrm{Li}, 2003)$. However, under many circumstances, the sample of spare parts consumption in a unit is small (Xu, 2013), so expanding the spare parts consumption data is an effective method to increase the fitting precision (Yang, 2012). In order to expand spare parts consumption data, this unit could draw on the experience of the pare parts consumption data in other units. It is essential to do research on the combination method of the pare parts consumption data (Mu, 2011).

Through analyzing the scientific researches on spare parts consumption, we could find that few papers have been published as of now regarding the combination of spare parts consumption data, which is formulated by combining spare parts consumption rules under both corrective maintenance and condition-based maintenance.

Based on the abstraction of above problems, a general solution is given to such kind of problems.

\section{COMBINATION OF SPARE PARTS CONSUMPTION DATA WITH TIME VARIATION}

At first, it is necessary to educe the reasonable environmental factor. Then according to the reasonable environmental factor, the spare parts consumption data in different environments can be transformed into the equivalent spare parts consumption data in the same environment. So the combination method of spare parts consumption data could be obtained in different environments.

\subsection{Environmental Factor}

Suppose the spare parts consumption rule of equipment unit is a distribution function concerning the parameter $t$, and the probability of spare parts consumption at the $t_{1}$ time is $F_{1}\left(t_{1}\right)$ in the first environment; the probability of spare parts consumption at the $t_{1}$ time is $F_{2}\left(t_{2}\right)$ in the second environment; the type of distribution function in the first environment is the same with that in the second environment; the probability of spare parts consumption at the $t_{1}$ time in the first environment is the same with that at the $t_{1}$ time in the second environment. The formula is (Liu, 2012)

$$
F_{1}\left(t_{1}\right)=F_{2}\left(t_{2}\right)
$$

If the type of distribution function of the spare parts consumption rule of equipment unit is normal distribution, i.e. $T_{1} \sim N\left(u_{1}, \sigma_{1}^{2}\right) ; T_{2} \sim N\left(u_{2}, \sigma_{2}^{2}\right)$, $u_{1}, \sigma_{1}^{2}, u_{2}, \sigma_{2}^{2}$ are respectively the average values and variances of $T_{1}$ and $T_{2}$. According to the formula (1),

$$
\Phi\left(\frac{t_{1}-u_{1}}{\sigma_{1}}\right)=\Phi\left(\frac{t_{2}-u_{2}}{\sigma_{2}}\right)
$$

According to the stochastic values $t_{1}$ and $t_{2}$, it is not hard to educe the relation between $T_{1}$ and $T_{2}$ (He, 2013),

$$
T_{1}=K_{N} T_{2}+B_{N}
$$

In the formula (3), $K_{N}=\frac{\sigma_{1}}{\sigma_{2}}$, and it is name is flex factor; $B_{N}=u_{1}-K_{N} u_{2}$, and it is name is translation factor. According to the formula (3), the conversion of the spare parts consumption data in the two different environments, is called the environmental factor method.

When the parameters of normal distribution in the two different environments are known, the environmental factor could be solved and it is a certain value. When the parameters of normal distribution in the two different environments are unknown, the estimation value of the environmental factor could be solved via the spare parts consumption data, then the estimation value of the environmental factor could be used to transform spare parts consumption data.

The distribution function of the environmental factor is also suitable for other types of distribution function of the spare parts consumption rule, for example, uniform distribution and triangle distribution. $K_{N}$ and $B_{N}$ are certain values via the parameters of distribution, and they are not stochastic values; $K_{N}$ and $B_{N}$ are all have 
functions of expression, and they are not hard to be calculated and used .

\subsection{Combination of Data}

Suppose the spare parts consumption data of equipment unit in the two different environments has been obtained.

The sample of spare parts consumption in the first environment is $z_{1}$, and the sample of spare parts consumption in the second environment is $z_{2}$.

When the parameters of the distribution in the two different environments are unknown, the conversion and combination of the spare parts consumption data are as follows:

(1) The maximum likelihood estimate method could be used to calculate the parameters of the distribution, then the point estimation values $K_{N}$ and $B_{N}$ could be calculated.

(2) According to the point estimation values $K_{N}$ and $B_{N}$, the spare parts consumption data in one environment can be transformed into the equivalent spare parts consumption data in another environment.

When the spare parts consumption data in the second environment is transformed into the data in the first environment, the formula is (Sheng, 2013)

$$
t_{1 j}^{\prime}=\hat{K}_{N} t_{2 j}+\hat{B}_{N} \quad j=1,2, \cdots, z_{2}
$$

(3) Composite the spare parts consumption data in the two different environments, the combination of the spare parts consumption data are

$$
t_{11}, t_{12}, \cdots, t_{1 z_{1}}, t_{11}^{\prime}, t_{12}^{\prime}, \cdots, t_{1 z_{2}}^{\prime}
$$

In this way, the sample of the spare parts consumption data of equipment unit could be expanded into $z_{1}+z_{2}$.

\section{COMBINATION OF SPARE PARTS CONSUMPTION DATA WITH YEARLY VARIATION}

\subsection{Combination of Homology Equipment Annual Consumption Data}

Composing the same variety of spare parts consumption data in many different environments could expand the sample of the spare parts consumption data of equipment unit and increase the forecasting precision of the spare parts consumption rule.

Suppose the spare parts consumption data comes from the same variety of equipment in $l$ environments, and the using conditions are the same, the number of the same variety of equipment units is $N$.

Suppose the number of the same variety of equipment is $n_{i}(i=1,2, \cdots, l)$, the number of spare parts consumption in the $i$ th environment in the $j$ th year is $x_{i j}(j=1,2, \ldots, m)$.

Statistics on homology equipment annual consumption in different environments is shown in Table 1.

Table 1: Statistics on homology equipment annual consumption in different environments

\begin{tabular}{|c|c|c|c|c|}
\hline Year & 1 & 2 & $\ldots$ & $m$ \\
\hline 1 & $x_{11}$ & $x_{12}$ & $\ldots$ & $x_{1 m}$ \\
\hline 2 & $x_{21}$ & $x_{22}$ & $\ldots$ & $x_{2 m}$ \\
\hline$\ldots$ & $\ldots$ & $\ldots$ & $\ldots$ & $\ldots$ \\
\hline$l$ & $x_{l 1}$ & $x_{l 2}$ & $\ldots$ & $x_{l m}$ \\
\hline Combination & $\frac{\sum_{i=1}^{l} x_{i 1}}{\sum_{i=1}^{l} n_{i} N}$ & $\frac{\sum_{i=1}^{l} x_{i 2}}{\sum_{i=1}^{l} n_{i} N}$ & $\ldots$ & $\frac{\sum_{i=1}^{l} x_{i m}}{\sum_{i=1}^{l} n_{i} N}$ \\
\hline
\end{tabular}

\subsection{Combination of Different Equipment Annual Consumption Data}

If the number of environments is very big, with regard to all the environments, it is essential to select the typical environments to do research on the combination of different equipment annual consumption data, then the spare parts consumption rule could be found.

Suppose the spare parts consumption data comes from the same variety of equipment in $l$ environments, and the using conditions are the same, the number of the units in the $k$ th equipment is $N_{k}$; the number of the $k$ th equipment in the $i$ th environment is $n_{k i}(i=1,2, \ldots, l, k=1,2, \ldots, q)$, the number of spare parts consumption in the $i$ th environment in the $j$ th year is $x_{i j}(j=1,2, \ldots, m)$.

Statistics on different equipment annual consumption in different environments is shown in Table 2.

Table 2: Statistics on different equipment annual consumption in different environments

\begin{tabular}{|c|c|c|c|c|}
\hline Year & 1 & 2 & $\ldots$ & $m$ \\
\hline 1 & $x_{11}$ & $x_{12}$ & $\ldots$ & $x_{1 m}$ \\
\hline 2 & $x_{21}$ & $x_{22}$ & $\ldots$ & $x_{2 m}$ \\
\hline$\ldots$ & $\ldots$ & $\ldots$ & $\ldots$ & $\ldots$ \\
\hline$l$ & $X_{l 1}$ & $x_{12}$ & $\ldots$ & $x_{l m}$ \\
\hline Combironment & $\sum_{i=1}^{l} x_{i 1}$ & $\frac{\sum_{i=1}^{l} x_{i 2}}{\sum_{i=1}^{l} \sum_{k=1}^{q} n_{k i} N_{k}}$ & $\ldots$ & $\frac{\sum_{i=1}^{l} x_{i m}}{\sum_{i=1}^{l} \sum_{k=1}^{q} n_{k i} n_{k i} N_{k}}$ \\
\hline
\end{tabular}




\section{CONCLUSIONS}

Nearly all the segments about aviation ammunition equipment maintenance materials include acquisition, storage, supplying and management have close connections with the aviation ammunition equipment maintenance materials consumption information.

The application of the aviation ammunition equipment maintenance materials consumption models based on the reliability of the units under conditionbased maintenance could be extended and the aviation ammunition equipment maintenance materials consumption models could also be improved aiming at solving different problems.

\section{ACKNOWLEDGEMENTS}

This This work is supported by the National Natural Science Fund of China (No.71401173).

\section{REFERENCES}

[1] He, S.Y., 2013. Stochastic Processes. Beijing, Peking University Press.

[2] Li S.P., Liu K.H., 2003. Optimized Dynamic Exponential Smoothing Model and Its Applications. Journal of Systems Engineering, vol. 18, pp.163-167.

[3] Liu J.K., Wang G.S., 2012. Applied Stochastic Processes. Beijing, Science Press.

[4] Mu H.L., Wang W.C., Ning Y.D., Li G., 2011. Study of Energy Consumption Prediction Based on Improved Grey Model. Journal of Dalian University of Technology, vol. 51, pp.493-497.

[5] Sheng Z., Xie S.Q., Pan C.Y., 2013. Probability Theory and Mathematical Statistics. Beijing, Higher Education Press.

[6] Xu D.J., 2013. A Further Study on the Exponential Smoothing Estimation Method for Parameters of Forecasting Model and Its Application. Systems Engineering-Theory \& Practice, vol. 2, pp. 25-30.

[7] Yang S.M., Guo J.S., Dong X.L., 2012. Combination Prediction Method for Aerial Spare Parts Based on LSSVM and Entropy. Fire Control \& Command Control, vol. 37, pp. 154-157. 